\title{
The metabolism of the ruminant cumulus-oocyte complex revisited
}

\author{
Jeremy G. Thompson, Robert B. Gilchrist ${ }^{1}$ and Melanie L. Sutton-McDowall \\ School of Paediatrics and Reproductive Health, The Robinson Institute and ARC Centre of Excellence in \\ Nanoscale BioPhotonics, The University of Adelaide, Adelaide 5005 SA AUSTRALIA; ${ }^{1}$ Current address: \\ School of Women's \& Children's Health, University of New South Wales, Sydney 2001, NSW AUSTRALIA
}

\begin{abstract}
Summary
The progress in understanding the metabolism of the ruminant cumulusoocyte complex (COC) from large antral follicles has progressed significantly in the past decade. In particular, new insights in the importance of lipid metabolism, $\beta$-oxidation and its relationship to oxidative phosphorylation within oocytes have emerged. This provides opportunities for tapping into the potential yield of ATP from lipid metabolism, as ATP content is a major determinant of oocyte competence. However, this new appreciation of lipid metabolism also includes the damaging influence of some free-fatty acids, the non-esterified fatty acids, which are particularly high in the follicular fluid of dairy cows during peak lactation. Historically, studies have focussed on carbohydrate metabolism, which occupies a central significance, especially for cumulus cell metabolism. Glucose has multiple roles; one still largely unexplored is its requirement for extracellular matrix production, via the hexosamine biosynthesis pathway. The availability of oxygen for oocytes in large antral follicles remains to be resolved, and requires new tools to measure intra-follicular $\mathrm{O}_{2}$ levels to determine its significance. For in vitro oocyte maturation, new strategies for improving oocyte competence includes: cAMP management, oocyte secreted factor supplementation, protection against reactive oxygen species with glutathione, and utilizing the endogenous maturation signalling molecules, the EGF-like peptides, during maturation - all of which influence metabolism. The introduction of existing platforms, such as metabolomics, to ruminant oocyte metabolism will broaden our understanding. Ultimately, to appreciate the dynamic nature of metabolism in oocytes of all species requires the development of new nano-scale sensing platforms that will allow us to measure activity in single oocytes and early embryos in situ in real time.
\end{abstract}




\section{Introduction}

In 2006, in chilly Wellington New Zealand, the Ruminant Reproduction Symposium VII was held within that country's spectacular national museum. One of the symposia talks at that meeting was on the same subject reviewed here. In the intervening 8 years there have been notable new insights into the metabolism of the ruminant cumulus-oocyte complex (COC), and so we have attempted to update our understanding in this review, with a strong focus on the bovine cumulus-oocyte complex.

Metabolomics allows comprehensive metabolic profiles to be created from relatively small amounts of sample (Koek, et al. 2010, Kraly, et al. 2009). This is particularly useful for the study of detailed amino acid or fatty acid profiles. Metabolomics involves the separation and then detection of metabolites; using technologies such as gas chromatography and high performance liquid chromatography for separation and mass spectrometry (MS), nuclear magnetic resonance (NMR) and Raman spectrometry for detection (Issaq, et al. 2009, Nagy, et al. 2009). Depending on the quality of sample, metabolomics can both identify and quantify (absolute concentration) or semi-quantify (relative changes between treatments) levels of substrates. Such metabolomic techniques have been restricted to human oocytes and embryos, confined to analysing either the embryo's secretome, or sampling the cumulus cells that are normally discarded, with the aim of selecting the best embryo for transfer (Nagy, et al. 2009, Nel-Themaat and Nagy 2011). Even though these techniques are capable of providing a comprehensive view of cellular metabolism, sampling over the time-course of oocyte maturation or embryo development is one major limitation in most metabolic studies, generally restricted to a single point in time. It remains that much of what we have learnt of ruminant COC metabolism in the ensuing eight years has still relied on the techniques of micro-sampling "spent" media and either direct analysis of media composition using fluorescence assays, or the application of fluorescence probes for oocytes. Some techniques, such as scanning electrode technology, has had limited application in ruminant oocytes (Sugimura, et al. 2012).

Interest in ruminant COC metabolism is driven by questions of how oocyte competence is regulated by the environment encountered during maturation. For example, there are concerns about oocyte quality within the dairy industry during peak lactation; interest grows in understanding the impact of environmental toxins on oocyte quality; and the emergence of a strong in vitro embryo production (IVP) market for cattle, and increasingly for sheep, goats, horses and pigs, has driven research to improve IVP efficiencies, with much of the focus on improving oocyte competence, as this is recognized as a bottleneck to IVP performance (Lonergan and Fair 2008, 2014). Such questions have strong links with mechanisms of cumulus cell and oocyte energy production and how metabolic activity is a determinant of oocyte competence.

\section{Oocyte developmental competency - what constitutes metabolic competence?}

Oocyte developmental competence is acquired in vivo as a result of complete follicular maturation achieved when the dominant follicle responds to the ovulatory surge of $\mathrm{LH}$, initiating a complex signalling cascade that impacts all follicular cells, including the oocyte. There are several transcriptomic studies comparing immature (hence not developmentally competent) to fully grown and ovulated oocytes, revealing major changes in the RNA processing and cell cycle control (e.g. Labrecque and Sirard 2014, Leoni, et al. 2007). Several studies have focussed on the cumulus cell transcriptome, in particular comparing in vivo matured vs. in vitro matured (IVM), in the attempt to understand why IVM-derived oocytes have poorer competence. Many of these studies reveal that metabolism of the COC is a key element in determining oocyte competence, with genes regulating both lipid and carbohydrate metabolism within cumulus cells being identified as associated with competence, (e.g. Peddinti, et al. 2010, Salhab, et al. 2013). 


\section{The cumulus-oocyte complex as a metabolic unit}

\section{Bi-directional communication in the COC}

The fundamental importance of the bi-directional communication between oocytes and their surrounding cellular vestment, the cumulus cell layer, is now a well-established paradigm in gamete biology. This relationship extends to metabolic co-operation as well and is the reason why, wherever possible, research involving the COC as an intact unit is increasingly favoured over the separation of these two distinct cellular types. Many review articles describe how the function of cumulus cells are significantly influenced by factors released from oocytes, primarily through the paracrine oocyte specific growth factors belonging to the TGF- $\beta$ family (for a review of ruminant oocyte factors, see Thompson, et al. 2012). In turn, the cumulus cells, when "healthily" maintained, provide nutrients, such as pyruvate and ATP to the oocyte (Buccione, et al. 1990) (Fig. 1). Immature oocytes at the GV stage of meiosis are connected with cumulus cell via a network of gap-junctions. Through junctional-complexes it is well established that nutrients and small molecular weight molecules pass from the cumulus cells to the oocyte (Anderson and Albertini 1976, Atef, et al. 2005, Buccione, et al. 1990, Sutovsky, et al. 1993) (Fig. 1). Gap-junctional communication is a determinant of oocyte competence, as either prolonging gap-junction communication enhances competence, or gap-junction disruption reduces competence following maturation (Luciano, et al. 2011, Luciano, et al. 2004, Thomas, et al. 2004). New insights into maintaining gap junction communication demonstrates the association with the maintenance of intra-oocyte cAMP levels (Luciano, et al. 2004) and impact of oocyte-secreted factors (Sugimura, et al. 2014). This most likely explains the positive benefit observed to oocyte competence following a brief pre-maturation period of high cAMP levels (e.g. Albuz, et al. 2010).

\section{Regulation of meiosis}

Prior to the ovulatory signal, oocytes are arrested at the germinal vesicle stage and this requires the production of cyclic adenosine monophosphate (cAMP) and cyclic guanidine monophosphate (cGMP), from cumulus cells and cAMP specifically in the oocyte. These levels are maintained by respective cyclases and phosphodiesterases (PDE). Initiation of the ovulatory surge leads to granulosa cell production of the EGF-like peptides (amphiregulin, epiregulin and betacellulin) that reduces CGMP levels, which in turn releases the inhibition of PDE type 3 within the oocyte, causing the hydrolysis of cAMP and release of meiosis. Recent new insights have demonstrated, at least in the mouse, how this elegant system of meiotic regulation is achieved (Conti, et al. 2012, Robinson, et al. 2012). From a metabolic perspective, there is a requirement for fentomole levels of $\mathrm{CGMP}$ and CAMP production. Energy is then required for chromatin reconfiguration and spindle formation. The picture in ruminants may differ in detail, but in essence the same or at least similar players are likely to operate. Yet differences do exist, for example, Sasseville, et al. (2009) has demonstrated that in cattle COCs, PDE8 is a dominant PDE, not present in the mouse.

\section{Intra-oocyte stores}

An area not well explored in mammalian oocyte biology is the storage of energy and mobilisation of stored energy. Somatic cells store energy in two forms: lipid and glycogen. Ruminant and porcine oocytes are distinctive for their high levels of lipid within oocytes. Lipids and their metabolism has been a recent focus of research, particularly in cattle oocytes (discussed 
elsewhere in more detail). Oocyte lipid content is highly variable across species. Recently, Krisher and colleagues (Paczkowski, et al. 2013) have proposed that the level of lipid reflects the time required from fertilisation to embryonic genome activation, being longer in species having lipid-rich oocytes. This suggests that there is some necessity for energy storage, a hypothesis supported by (Cetica, et al. 2003). In contrast, there is very little known about glycogen synthesis or storage in COCs. There is a paucity of literature on this subject, even in the mouse (Pike and Wales 1982, Thompson, et al. 1995). This is not the case for amphibian and drosophila oocytes, where glycogen has important functions in meiotic control, (e.g. Dworkin and Dworkin-Rastl 1989, Gutzeit, et al. 1994). Whether it is present and what role it plays in ruminant oocytes has yet to be determined.

\section{ATP production is what it's all about?}

ATP production is the essential purpose for metabolism, yet many studies (including our own) do not always measure ATP (and ADP) levels. Many studies infer differences in ATP production by defacto measurements such as mitochondrial activity and/or oxygen consumption (e.g. Sugimura, et al. 2012). Furthermore, if measured, most studies determine ATP levels at single points during oocyte maturation (e.g. Stojkovic, et al. 2001). Furthermore, measurement of the ATP:ADP ratio, a measure of ATP turnover as a consequence of demand, is rarely reported.

Apart from the provision of energy within the oocyte, ATP is the substrate used by adenylate cyclase for cAMP generation, essential for maintaining meiotic arrest at GV stage in oocytes. New approaches for measuring ATP applied to (mouse) oocyte maturation has revealed that peaks in demand within the oocyte is closely linked with stages of chromatin remodelling during meiotic progression (Dalton, et al. 2014). Significantly, oocyte competence is highly correlated to ATP levels (Dalton, et al. 2014, Stojkovic, et al. 2001). Even more significantly, maintaining gap-junctional communication supports higher levels of ATP within the oocyte, validating this approach for improving oocyte competence (Dalton, et al. 2014). Nevertheless, cumulus cell and oocyte metabolism is more than simply the need for oocyte ATP production, for example, the production of cumulus-derived matrix and intra-oocyte glutathione are both examples of alternate metabolic requirements.

\section{Glucose metabolic pathways}

Glucose is an essential requirement for the bovine cumulus-oocyte complex for both meiotic and cytoplasmic maturation. A well-founded principle for mammalian species, including cattle, is that the cumulus-oocyte complex consumes high levels of glucose, which is metabolized by the cumulus cells through primarily the glycolytic pathway (Cetica, et al. 2002) (Fig. 1). In contrast, the oocyte itself has a low capacity for glucose metabolism, and is reliant on ATP production from oxidative phosphorylation. This low capacity for glucose oxidation in oocytes was recognized in cattle many decades ago (e.g. Rieger and Loskutoff 1994, Rushmer and Brinster 1973). Although attributed to inhibition of phosphofructokinase (Barbehenn, et al. 1974), we remain ignorant as to the advantage there is for an oocyte to be reliant on carboxylic acids supplied to it from cumulus cells, rather than having its own capacity to do so. 
Glycolysis

Glycolysis is the primary glucose metabolic pathway within the cumulus cells of the COC, and is essential for mammalian (including cattle) maturation. Inhibition of glycolytic activity in cattle COCs impacts negatively on both oocyte meiotic maturation and mitochondrial activity (Gutnisky, et al. 2013b). Varying the level of glucose availability also influences meiotic regulation, presumably by a change in glycolytic flux through cumulus cells, impacting oocyte meiotic regulation (Sutton-McDowall, et al. 2005). Even though the oocyte has only a limited capacity for intra-oocyte glycolysis, the amount that is metabolised through this pathway has been positively associated with competence (Krisher 2013, Krisher and Bavister 1999). This suggests an as yet un-identified process that requires some degree of oocyte glycolysis.

A concept that first emerged in the 1990's (Rieger 1992) and now revisited is the parallel metabolic profiles of pre-implantation embryos and cancer cells (Krisher and Prather 2012, Smith and Sturmey 2013). Like some cancer cells and other cell types that undergo active proliferation, cumulus cells and post-compaction embryos utilize a large amount of glucose via aerobic glycolysis, resulting in significant levels of lactate production, in preference to a flux of pyruvate through the TCA cycle and oxidative phosphorylation, a phenomenon called the Warburg effect (Warburg 1956). In cancer cells, this occurs despite glycolysis being relatively inefficient at producing ATP compared to oxidative phosphorylation (2 ATP vs. 38 ATP), even though a large demand for ATP to fuel rapid cell division exists. Krisher and Prather (2012) suggest that aerobic glycolysis provide the embryo with substrates for cell division such as nucleic acid synthesis (pentose phosphate pathway, PPP) and reduction-oxidation equilibrium (NADPH and $\mathrm{NADH}$ ), with other metabolites such as fatty acids and amino acids, providing substrates for oxidative phosphorylation and ATP. This is primarily achieved through the expression of enzymes, such as pyruvate kinase M2 (PKM2) that has low kinase activity, resulting the in the build-up of glycolytic intermediates and preferential metabolism via PPP and the hexosamine biosynthetic pathway (HBP). Confirmation that the Warburg effect applies to cumulus cells within the COC have yet to be established, but it is an attractive framework for understanding cumulus cell metabolism. In 2007 (Thompson, et al. 2007), we hypothesized that one function of the bi-directional communication between oocytes and cumulus cells was to establish a metabolic relationship between the oxidative metabolism needs of the oocyte and the glycolytic activity of the cumulus. In the mouse, it is well established that OSFs from the oocyte regulate cumulus cell glycolytic activity (Sugiura and Eppig 2005, Sugiura, et al. 2005, Sugiura, et al. 2007). Using mathematical modelling, we determined that relative to oocytes, on a per volume of tissue basis, bovine cumulus cells consume 20-fold more glucose than oocytes (Clark, et al. 2006, Clark, et al. 2011). This led us to examine by mathematical modelling, the relationship between follicular glucose content and the intra-oocyte cortical concentration of glucose, assuming that a glucose concentration gradient across the cumulus cells was established by diffusion (Stokes, et al. 2008). Modelling demonstrated a significant glucose gradient across the cumulus layer such that even a modest change (e.g. $1.5 \mathrm{mM}$ ) in follicular concentration of glucose made a substantial difference in the concentration at the boundary between the cumulus layer and oocyte. Further work on modelling the differences in glucose uptake between the intact COC and mural granulosa cells revealed a 4- to 6-fold higher uptake by cumulus cells, suggesting bovine COC glucose uptake is probably under the regulation of OSFs (Clark, et al. 2011). This differs from our original observation that oocytectomy did not alter glucose uptake (Sutton, et al. 2003). The discrepancy is almost certainly due to the impact of supplemental FSH in our original work, as we have seen that FSH has an overriding effect on metabolism in other studies, for example the influence of the OSF, BMP-15 (Sutton-McDowall, et al. 2012). A recent finding is that substituting FSH with 
the EGF-like peptide amphiregulin, preserves the impact of BMP-15 on several metabolic measures, and co-operatively enhances developmental competence (Sugimura, et al. 2014), therefore acting differently to $\mathrm{FSH}$.

\section{The Hexosamine Biosynthesis Pathway}

It is well established that both glucose and glutamine are essential substrates for the hexosamine biosynthesis pathway (HBP), required for the formation of hyaluronic acid and cumulus expansion during IVM (Frank, et al. 2014, Furnus, et al. 1998, Sutton-McDowall, et al. 2010). Prior to a cumulus matrix expansion signal (such the LH surge in vivo, or incubation with $\mathrm{FSH}$ in vitro), the activity of this pathway accounts for little glucose consumed by the COC (Gutnisky, et al. 2007, Sutton-McDowall, et al. 2004). The activity of this pathway is greatly activated by the cumulus expansion signal (Fig. 1), yet the signalling pathway regulating this is still unexplored in mammalian species to date, although candidates should include the Akt-mTOR pathway (Chen, et al. 2013) or ERK (Richani, et al. 2013).

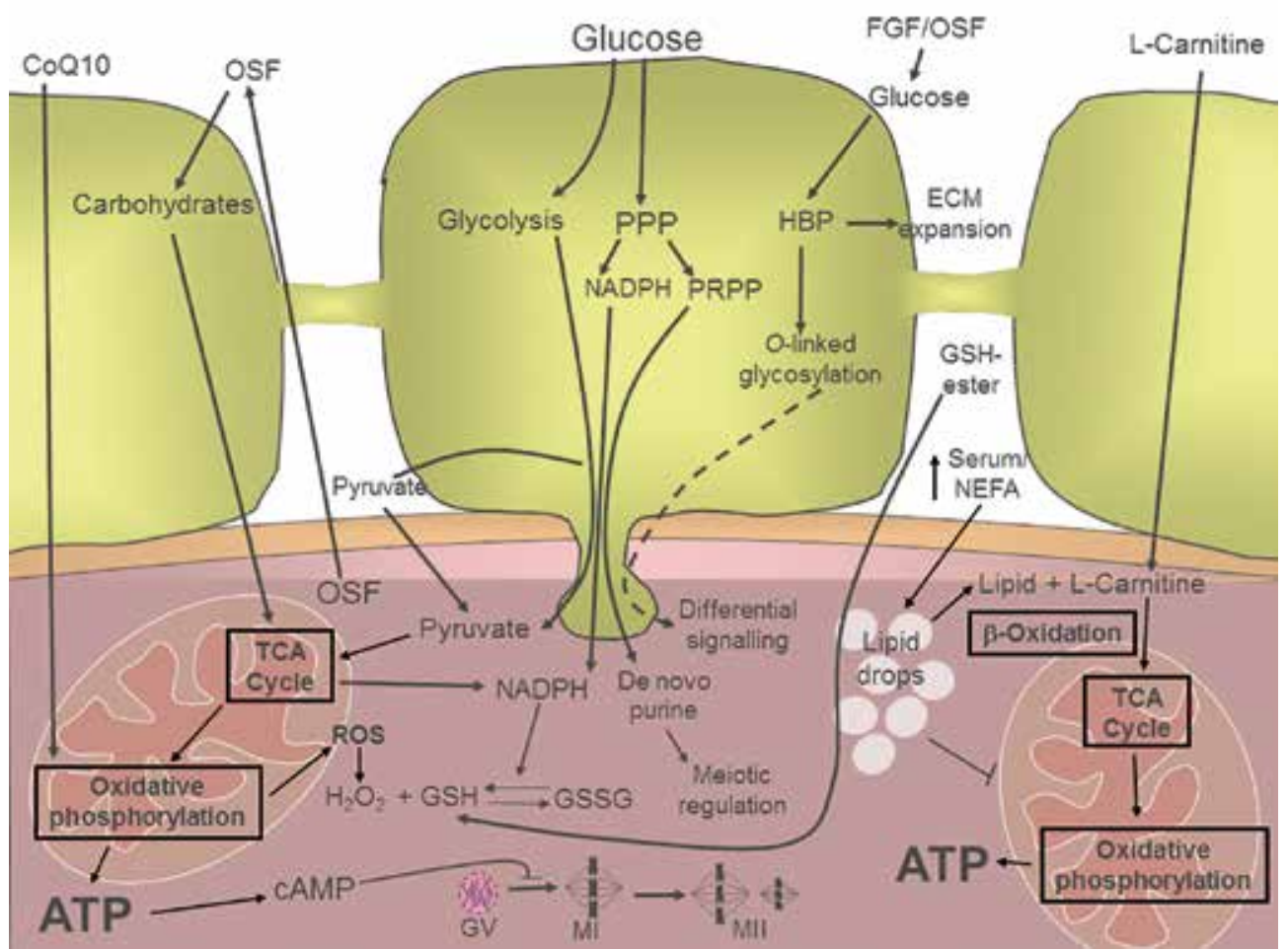

Fig. 1. Metabolism of the intact cumulus oocyte complex (COC). Carbohydrates, in particular glucose, are a primary energy source of energy and substrates for signalling. Glucose is metabolised by the cumulus cells via glycolysis (energy/pyruvate production), pentose phosphate pathway (PPP; NADPH production and meiotic regulation) and the hexosamine biosynthetic pathway (HBP; signalling and extra cellular matrix, ECM, synthesis). Within the oocyte, the tricarboxylic acid (TCA) cycle and oxidative phosphorylation are important for metabolising pyruvate and endogenous lipids following $\beta$-oxidation, within the mitochondria. Endogenous anti-oxidants such as glutathione (reduced $=\mathrm{GSH}$, oxidised $=$ GSSG) are extremely important for combating reactive oxygen species (ROS), such as $\mathrm{H}_{2} \mathrm{O}_{2}$, a product of oxidative phosphorylation. There are numerous emerging factors that influence $\mathrm{COC}$ metabolism, such as oocyte secreted factors (OSF), fibroblast growth factor (FGF), co-enzyme Q10 (CoQ10) and L-carnitine. 
Recently, (Caixeta, et al. 2013) demonstrated that mRNA levels for the HBP rate limiting enzymes, glutamine:fructose-6-phosphate transaminase (GFPT) 1 and 2 are up-regulated by either BMP-15 or FGF-10, with both these growth-factors stimulating a 30\% increase in glucose uptake without a concomitant rise in lactate production, suggestive of an increased flux through the HBP.

Our previous review (Thompson, et al. 2007) pointed to the likely inhibitory consequences for post-compaction embryo development from an up-regulated HBP during oocyte maturation. This involves the participation of an "energy-sensing" pathway, $\beta$-O-linked glycosylation (O-GlcNAc), and its impact on intracellular signalling (reviewed by Frank, et al. 2014). This pathway is intimately involved in glucose sensing and heavily implicated in the pathology of diabetes (Frank, et al. 2014). Although our subsequent studies have been conducted in the mouse, it is pertinent to recall that the original observations were made in cattle and pigs (Sutton-McDowall, et al. 2006). In dairy cattle, oocyte quality is more likely to be affected by hypoglycaemic conditions. This too can implicate the HBP and O-GlcNAc (Frank, et al. 2013), yet there are no studies investigating this in ruminant oocytes.

\section{Pentose Phosphate Pathway}

There is increased interest in the Pentose Phosphate Pathway (PPP) as a result of the application of a relatively simple assay (the brilliant cresyl blue, or BCB assay) that measures activity for the pathway's rate-limiting enzyme, glucose-6-phosphate dehydrogenase (G-6-PDH). First described for use in GVstage pig oocytes (Roca, et al. 1998), it has rapidly become a test for immature oocyte competence e.g. (Castaneda, et al. 2013, Pujol, et al. 2004). On addition of BCB, when G-6-PDH is active, the cytoplasm of GV stage oocytes turn from a deep blue colour to transparent. This is associated with poor cytoplasmic maturity. In contrast, oocytes remaining deep blue are more competent following maturation. Interestingly, this assay has overwhelmingly been used prior to oocyte maturation as an assessment of GV-stage oocyte development. However, PPP activity has been associated with meiotic and developmental competence of mature oocytes in the mouse and cow (Downs, et al. 1998, Gutnisky, et al. 2013a). Despite this, there are few studies investigating post-maturation use of the BCB assay. The exception is Mirshamsi, et al. (2013), who observed that whereas competent GV-oocytes stained blue, competent zygotes had clear cytoplasm, suggesting an increase in PPP activity over the course of maturation and fertilization.

\section{Oxygen supply to the follicle and oocyte oxidative phosphorylation}

An intriguing aspect of oocyte energetics is the oocyte's absolute requirement for oxidative phosphorylation (Fig. 1). This has been well established in several model species, including mouse and cow (Rushmer and Brinster 1973, Thomson 1967). Pyruvate is the preferred carboxylic acid, feeding into the tricarboxylic acid cycle. The $\mathrm{O}_{2}$ consumption of the oocyte (per $\mathrm{ml}$ of tissue) is 3-fold higher than cumulus cells (Clark, et al. 2006), even though it would appear if cumulus does have a significant oxidative capacity (Cetica, et al. 2003). Further modelling, based on actual levels of $\mathrm{O}_{2}$ consumption by whole COCs (Sutton, et al. 2003), suggest that the decrease in $\mathrm{O}_{2}$ concentration at the cortex of the oocyte is approximately $3-4 \mathrm{mmHg}$ (about $0.5 \%$ ) less than within the follicular antrum, over a physiological range of antral $\mathrm{pO}_{2}$ (Clark, et al. 2006). Therefore, relatively little $\mathrm{O}_{2}$ is consumed by the cumulus layer, ensuring that the oocyte avoids hypoxia. The intrigue referred to above is that within large antral follicles, the oocyte lies within a highly avascular environment, many cell layers thick. Despite the publication by Van Blerkom (Van Blerkom, et al. 1997) on human follicles remaining a standout in this area, the question of whether follicular $\mathrm{pO}_{2}$ regulates oocyte competence remains poorly addressed, largely because of the technical difficulties that such measurements present. 
Using an alternative mathematical modelling approach, Redding and colleagues (Redding, et al. 2007, 2008) rationalised the formation of the follicular antrum as a response to $\mathrm{O}_{2}$ and nutrient demand. Their conclusion is that formation of an expanding antrum allows cellular proliferation to occur without adding to the number of avascular cell layers surrounding the oocyte. As a consequence, they predicted that antral $\mathrm{pO}_{2}$ would reflect the thickness of layers of mural granulosa and cumulus cells. In doing so, they predicted the human antral follicle $\mathrm{pO}_{2}$ was quite variable, around 1.5 $6.7 \% \mathrm{O}_{2}$ (11-51 $\mathrm{mmHg}$ ); this appears similar to their data for cattle follicles (Redding, et al. 2006). At such concentrations, it is feasible that hypoxic response mechanisms occur, but there has been very little work conducted on mechanisms such as activation of the hypoxia inducible factor family of transcription factors in oocytes and follicles, with much of what is known being focussed on rodent models (Alam, et al. 2004, Alam, et al. 2009, Kind, et al. 2014).

Within the oocyte, mitochondrial activity and localisation reflect oocyte competence (Hashimoto 2009, Sugimura, et al. 2012). Highly competent ovine oocytes are associated with a peri-cortical and peri-nuclear mitochondrial distribution (Martino, et al. 2012). Generation of reactive oxygen species (ROS) is a normal consequence of oxidative phosphorylation within functioning mitochondria (Martino, et al. 2012, Morado, et al. 2009). Oxidative bursts within fertilizing mouse and cow oocytes are associated with chromatin reconfiguration (Dumollard, et al. 2004, Morado, et al. 2013). Morado and colleagues (Morado, et al. 2009) measured the changes in ROS patterns over the timecourse of IVM and observed that ROS levels decrease between 6-18 hr maturation, coinciding with meiotic reconfiguration, usually at their lowest by $12 \mathrm{~h}(\mathrm{Ml})$ in cattle oocytes. Against current dogma, these authors saw little association between ROS levels and inhibition or stimulation of oxidative phosphorylation, but observed a significant reduction with the addition of cysteine, an essential amino acid required for glutathione (GSH) synthesis. Much has been written about the requirement for antioxidant therapy, both during IVM of oocytes and subsequent embryo culture, yet most therapies applied in vitro yield disappointing results. Reduced GSH is a well characterised antioxidant and increasing intra-oocyte concentrations is known to be associated with improved oocyte competence in several ruminant species (Curnow, et al. 2010, de Matos, et al. 1995, de Matos, et al. 2002) (Fig. 1). Strategies to increase intra-oocyte GSH levels have involved the supplementation to medium of cysteine, usually in the presence of another reduced thiol compound, such as cysteamine or $\beta$-mercaptoethanol (de Matos and Furnus 2000). Cumulus cell GSH production from thiols such as cysteine and cysteamine appears to be important for the oocyte (de Matos, et al. 1997). An alternative that has yet to be fully exploited is the use of a GSH-ester, which has been demonstrated to increase intra-oocyte GSH levels in both cumulus-enclosed and denuded oocytes (Curnow, et al. 2010). Another powerful antioxidant that is generating interest is co-enzyme Q10 (CoQ10). CoQ10 is an essential component of the respiratory chain, and is widely used as an antioxidant dietary supplement. Supplementation of CoQ10 to bovine oocytes during in vitro maturation has been associated with increased ATP production (Stojkovic, et al. 1999) and can impact mitochondrial localisation and membrane potential, as well as gene expression and overall competence in a compromised oocyte model (Gendelman and Roth 2012).

\section{The new insight in ruminant COC metabolism - lipid metabolism}

At the time of writing the 2007 review (Thompson, et al. 2007), there was very little known regarding lipid metabolism in mammalian oocytes. In the intervening 8 years, this is where the greatest new insights have been obtained in the field, with wide implications for the fertility of cattle and the development of ruminant in vitro media systems.

Ruminant oocytes and cumulus cells are particularly lipid rich, containing considerably higher levels than human and mouse. For example, sheep and cow oocytes contain $21 \mathrm{ng} / \mathrm{ml}$ 
and $15 \mathrm{ng} / \mathrm{ml}$ w/w lipids, respectively, compared with $6.3 \mathrm{ng} / \mathrm{ml}$ in mouse oocytes (reviewed by Sturmey, et al. 2009). The "time to attachment" hypothesis (Paczkowski, et al. 2013, Sturmey, et al. 2009), suggests the period between ovulation and implantation dictates lipid content; there is 4- 6 days between ovulation and implantation in the mouse verses 15-30 days in sheep and cattle.

Triglycerides are the predominant lipid found within the oocyte, with palmitic acid and oleic acid accounting for $32 \%$ and $25 \% \mathrm{w} / \mathrm{w}$ of total lipids within cow oocytes and $25 \%$ and $26 \%$ w/w within sheep oocytes (McEvoy, et al. 2000a). While lipids are important substrates for steroid hormone, prostaglandins and membrane biosynthesis, lipids are also a valuable energy source. Lipid-derived fatty acids are metabolised to acetyl CoA via $\beta$-oxidation and when completely metabolised, $1 \mathrm{M}$ of lipid (such as palmitic acid) will yield $\sim 106 \mathrm{M} \mathrm{ATP}$, compared to 27-31 M ATP from $1 \mathrm{M}$ of oxidised glucose. Indeed, in the absence of carbohydrates, the addition of L-carnitine (a co-factor of carnitine palmitoyl transferase-I, the rate-limiting enzyme in $\beta$-oxidation (Dunning and Robker 2012)) assists in the mobilisation of endogenous lipid stores, capable of supporting pre-compaction embryo development similar to rates achieved in the presence of carbohydrates (Sutton-McDowall, et al. 2012) (Fig. 2). Conversely, inhibiting $\beta$-oxidation during maturation and early embryo development significantly perturbs oxygen consumption and blastocyst development (Ferguson and Leese 2006).

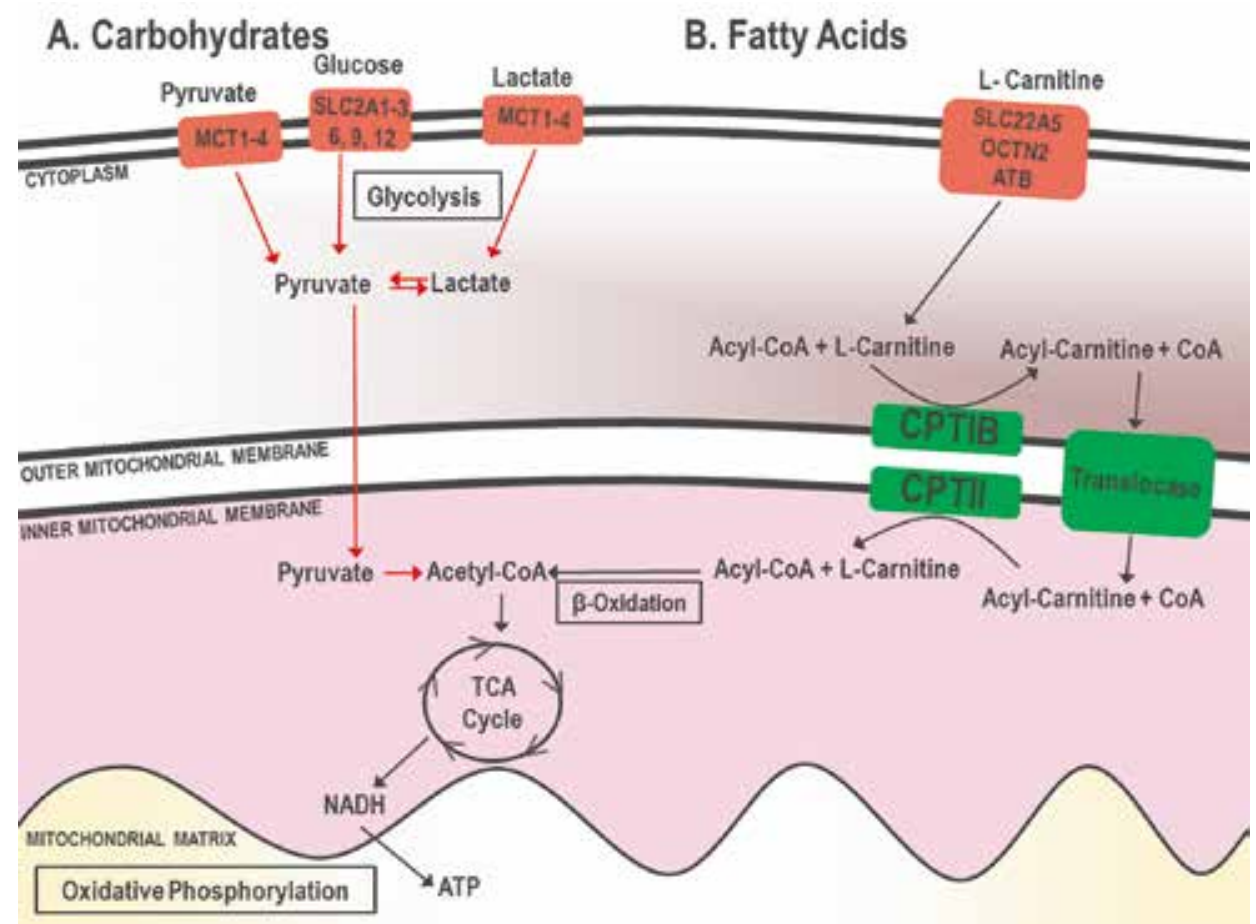

Fig. 2. Both A) carbohydrates and B) endogenous fatty acids can be utilised for energy production within the mitochondria, via the tricarboxylic acid (TCA) cycle and oxidative phosphorylation. MCT $1-4=$ monocarboxylate transporters 1-4 (pyruvate/lactate); SLC2A 1-3, 6, 9, 12 = Solute carrier family 2, facilitated glucose transporter members (glucose); SLC22A5/OCTN2 = Solute carrier family 22 (organic cation/ carnitine transporter), member 5 (L-carnitine); ATB = amino acid/carnitine transporter B (L-carnitine); $\mathrm{CPTIB} / \mathrm{CPTIIB}=$ carnitine palmitoyltransferase. Adapted from (Sutton-McDowall, et al. 2012). 
Despite the importance of lipids as an endogenous energy source, increased lipid accumulation within COCs is associated with compromised developmental competence and fetal outcomes, such as large calf syndrome (Leese, et al. 1998, McEvoy, et al. 2000b). In vitro serum supplementation during cow and sheep IVP was identified as a major cause (reviewed by Young, et al. 1998). Increased lipid content is associated with poor cryo-tolerance of embryos, possibly due to poor lipid metabolism in vitro and the absence of L-carnitine in culture systems. When added during IVM, L-carnitine improved the cryo-tolerance of cattle COCs, resulting in similar on-time embryo development as un-vitrified oocytes following IVF and IVC (Chankitisakul, et al. 2013, Phongnimitr, et al. 2013). Both these studies used serum as a protein source, indicating that decreasing the serum-induced accumulation of lipid maybe the greatest benefit of L-carnitine under these conditions. It has yet to be determined if L-carnitine supplementation of medium containing either BSA or no protein results in increased developmental competence or cryo-tolerance.

\section{Non-esterified fatty acids and dairy cow fertility}

The increasing rate of sub-fertility in high performance, milk-yielding cows has been partially attributed to increased accumulation of fatty acids by the COC, in particular non-esterified fatty acids (NEFAs). NEFAs are long-chain (greater than 14 carbons) free fatty acids that are distinguished from triacylglycerides (esterified fatty acids). Many farming systems involve a 12-month calving interval, requiring cows to be pregnant by 60-80 days post-partum, coinciding with the peak lactation period (0-70 days post-partum). During peak lactation, metabolites (for example, up to $80 \%$ of glucose) are partitioned for lactation and away from other organs. As a consequence, body condition scores decrease and cows enter negative energy balance (NEB) (Bell, et al. 1995, Hocquette and Bauchart 1999, Walsh, et al. 2011). A consequence of NEB is the mobilisation of fats, in particular NEFAs, with the severity of NEB directly related to increases in circulating NEFA levels (Schneider 2004). Considering the final stages of oocyte development and maturation commence 90 days prior to ovulation (Fair 2010), oocyte developmental competence could be compromised, resulting in poor fertilisation, embryo development and early pregnancy losses. This led MacMillan (2007) to describe these cows as suffering from "Phantom Cow Syndrome", where cows fail to return to oestrus by the second round of artificial insemination, indicative of successful fertilisation but subsequent embryonic loss.

A cause of sub-fertility in high milk yielding dairy cattle that has recently gained attention is the influence of the peak lactation on the follicular environment. Levels of specific NEFAs, namely the saturated fatty acids palmitic (C16:0) and steric acid (C18:0) and the mono-unsaturated oleic acid (C18:1) are higher in follicular fluid (FF) compared with serum from 16 day post-partum cows (Leroy, et al. 2005), but returned to serum levels by 44 days post-partum. In addition, $\beta$-hydroxybutyrate levels at Day 16 post-partum mirror the elevated NEFA concentrations, further suggesting mobilisation of body reserves (Matoba, et al. 2012). When NEFAs were added to IVM culture of COCs at levels equivalent of Day 16 FF, bovine oocytes had more and larger lipid droplets, a reduced blastocyst yield and resultant blastocysts had less cells, higher rates of apoptosis and altered metabolism (Aardema, et al. 2011, Van Hoeck, et al. 2011). Gene expression of glucose metabolic enzymes were also influenced, with higher levels of lactate dehydrogenase (LDH1) and glyceraldehyde 3-phosphate dehydrogenase (GADPH) observed in oocytes from COCs exposed to high NEFAs. Furthermore, these genes, in addition to glucose-6-phosphate dehydrogenase $(\mathrm{G} 6 \mathrm{PDH})$ were lower in cumulus cells (Van Hoeck, et al. 2013). Given the majority of glucose consumed by the COC is metabolised via glycolysis 
within cumulus cells (Sutton-McDowall, et al. 2010), this suggests the presence of elevated NEFAs decreases glucose metabolism.

\section{Insights from "metabolomics" studies in ruminants}

To date, there are minimal metabolomic studies published investigating ruminant oocytes. Most studies have focused on human clinical oocyte and embryo development (Nagy, et al. 2009, Singh and Sinclair 2007). However, Bender and colleagues (Bender, et al. 2010) have successfully used gas chromatography-mass spectrometry (GC-MS) to analyse the different fatty acid and amino acid profiles of FF derived from Holstein heifers ("good") vs. lactating cows ("compromised"). Thirty seven fatty acids were detected (25 quantifiable) and 52 aqueous metabolites were detected (20 quantifiable) (Bender, et al. 2010). The predominant fatty acids reported here were similar to other reports (Leroy, et al. 2005). Matrix-assisted laser desorption ionization (MALDI) mass spectrometry has recently been used to analyse lipid content in oocytes and embryos of several species, including cattle (Ferreira, et al. 2010), revealing compositional differences under different $\mathrm{O}_{2}$ atmospheres and between in vivo and in vitro derived oocytes. Analysis of amino acids and aqueous metabolites revealed L-alanine was lower and glycine, glutamine and urea were higher in the FF of cows compared to heifers. Metabolomics has also been utilised to determine the amino acid turnover by bovine oocytes during IVM. This study analysed amino acid metabolism of denuded oocytes between 18 - $24 \mathrm{hr}$ IVM, comparing the spent medium profile from oocytes that had undergone cleavage and development to the blastocyst stage against unfertilised oocytes (Hemmings, et al. 2012). Oocytes that did not cleave had less glutamine and more alanine in the surrounding medium than successfully cleaving and developing oocytes. The similarity in specific non-essential amino acids that were altered between these two studies may not be a coincidence, but if so, the relationship is not clear.

\section{The future of metabolic profiling of ruminant oocytes}

Cattle COC metabolism is now characterised sufficiently to answer questions about differences between the effectiveness of in vitro media composition and the impact of maternal environments (such as differences between lactating dairy cattle and heifers). The number of parameters that can be measured is increasing, including reliable measures of the REDOX state, reduced glutathione, ATP/ADP and reactive oxygen species. We are gaining an understanding of how metabolism is facilitated by factors that influence competence, such as the impact of gapjunctional communication, OSFs and EGF-like peptides. What we have learnt is that there is enormous plasticity in metabolic regulation. Simply measuring one metabolic pathway or intermediate metabolite may not reconcile with developmental outcome. Therefore the future direction of metabolic research requires a more holistic and expansive determination of metabolism and this is where true metabolomic studies will be an important element. Nevertheless, endpoints of metabolism, such as ATP and reduced glutathione levels are at least indicative measures of competence.

In many aspects, our tools that have been applied to date to study ruminant oocyte metabolism have reached the limits of their capability. What is really required is the capacity to observe the dynamic nature of metabolism over a developmental period, such as the period of oocyte maturation. There are emerging tools to measure metabolism non-destructively over time, such as time-lapse incubation confocal microscopy. In addition to this, microscopic advances, especially in measuring fluorescence (whether it be autofluorescence or cell compatable 
fluorophores) at unprecedented scales of resolution are being rapidly developed. New capabilities in photonics and nano-particle sensing offer opportunities to measure the dynamic nature of early developmental events within single oocytes and embryos, potentially while in situ.

\section{References}

Aardema, H, PL Vos, F Lolicato, BA Roelen, HM Knijn, AB Vaandrager, JB Helms, and BM Gadella 2011 Oleic acid prevents detrimental effects of saturated fatty acids on bovine oocyte developmental competence. Biol Reprod 85 62-69.

Alam, H, ET Maizels, Y Park, S Ghaey, ZJ Feiger, NS Chandel, and M Hunzicker-Dunn 2004 Folliclestimulating hormone activation of hypoxia-inducible factor- 1 by the phosphatidylinositol 3-kinase/AKT/Ras homolog enriched in brain (Rheb)/mammalian target of rapamycin (mTOR) pathway is necessary for induction of select protein markers of follicular differentiation. J Biol Chem 279 19431-19440.

Alam, H, J Weck, E Maizels, Y Park, EJ Lee, M Ashcroft, and M Hunzicker-Dunn 2009 Role of the phosphatidylinositol3-kinase and extracellular regulated kinase pathways in the induction of hypoxia-inducible factor (HIF)-1 activity and the HIF-1 target vascular endothelial growth factor in ovarian granulosa cells in response to follicle-stimulating hormone. Endocrinology 150 915-928.

Albuz, FK, M Sasseville, M Lane, DT Armstrong, JG Thompson, and RB Gilchrist 2010 Simulated physiological oocyte maturation (SPOM): a novel in vitro maturation system that substantially improves embryo yield and pregnancy outcomes. Hum Reprod 25 2999-3011.

Anderson, E, and DF Albertini 1976 Gap junctions between the oocyte and companion follicle cells in the mammalian ovary. J Cell Biol 71 680-686.

Atef, A, P Francois, V Christian, and M-A Sirard 2005 The Potential Role of Gap Junction Communication Between Cumulus Cells and Bovine Oocytes During in Vitro Maturation. Mol Reprod Dev 71 358-367.

Barbehenn, EK, RG Wales, and OH Lowry 1974 The explanation for the blockade of glycolysis in early mouse embryos. Proc Natl Acad Sci U S A 71 1056-1060.

Bell, AW, R Slepetis, and RA Ehrhardt 1995 Growth and accretion of energy and protein in the gravid uterus during late pregnancy in Holstein cows. J Dairy Sci 78 1954-1961.

Bender, K, S Walsh, AC Evans, T Fair, and L Brennan 2010 Metabolite concentrations in follicular fluid may explain differences in fertility between heifers and lactating cows. Reproduction 139 1047-1055.

Buccione, R, AC Schroeder, and JJ Eppig 1990 Interactions between somatic cells and germ cells throughout mammalian oogenesis. Biol Reprod 43 543-547.

Caixeta, ES, ML Sutton-McDowall, RB Gilchrist, JG Thompson, CA Price, MF Machado, PF Lima, and J Buratini 2013 Bone morphogenetic protein 15 and fibroblast growth factor 10 enhance cumulus expansion, glucose uptake, and expression of genes in the ovulatory cascade during in vitro maturation of bovine cumulusoocyte complexes. Reproduction 146 27-35.

Castaneda, CA, P Kaye, M Pantaleon, N Phillips, S Norman, R Fry, and MJ D'Occhio 2013 Lipid content, active mitochondria and brilliant cresyl blue staining in bovine oocytes. Theriogenology 79 417-422.

Cetica, P, L Pintos, G Dalvit, and M Beconi 2002 Activity of key enzymes involved in glucose and triglyceride catabolism during bovine oocyte maturation in vitro. Reproduction 124 675-681.

Cetica, P, L Pintos, G Dalvit, and M Beconi 2003 Involvement of enzymes of amino acid metabolism and tricarboxylic acid cycle in bovine oocyte maturation in vitro. Reproduction 126 753-763.

Chankitisakul, V, T Somfai, Y Inaba, M Techakumphu, and T Nagai 2013 Supplementation of maturation medium with L-carnitine improves cryo-tolerance of bovine in vitro matured oocytes. Theriogenology 79 590-598.

Chen, J, S Torcia, F Xie, CJ Lin, H Cakmak, F Franciosi, K Horner, C Onodera, JS Song, MI Cedars, M RamalhoSantos, and M Conti 2013 Somatic cells regulate maternal mRNA translation and developmental competence of mouse oocytes. Nat Cell Biol 15 1415-1423.

Clark, AR, YM Stokes, M Lane, and JG Thompson 2006 Mathematical modelling of oxygen concentration in bovine and murine cumulus-oocyte complexes. Reproduction 131 999-1006.

Clark, AR, YM Stokes, and JG Thompson 2011 Estimation of glucose uptake by ovarian follicular cells. Ann Biomed Eng 39 2654-2667.

Conti, M, M Hsieh, AM Zamah, and JS Oh 2012 Novel signaling mechanisms in the ovary during oocyte maturation and ovulation. Mol Cell Endocrinol 356 65-73.

Curnow, EC, JP Ryan, DM Saunders, and ES Hayes 2010 Developmental potential of bovine oocytes following IVM in the presence of glutathione ethyl ester. Reprod Fertil Dev 22 597-605.

Dalton, CM, G Szabadkai, and J Carroll 2014 Measurement of ATP in single oocytes: impact of maturation and cumulus cells on levels and consumption. J Cell Physiol 229 353-361.

de Matos, DG, and CC Furnus 2000 The importance of having high glutathione (GSH) level after bovine in vitro maturation on embryo development effect of betamercaptoethanol, cysteine and cystine. Theriogenology 53 761-771.

de Matos, DG, CC Furnus, and DF Moses 1997 Glutathione synthesis during in vitro maturation of bovine oocytes: role of cumulus cells. Biol Reprod 57 1420-1425. 
de Matos, DG, CC Furnus, DF Moses, and H Baldassarre 1995 Effect of cysteamine on glutathione level and developmental capacity of bovine oocyte matured in vitro. Mol Reproduction Dev 42 432-436.

de Matos, DG, B Gasparrini, SR Pasqualini, and JG Thompson 2002 Effect of glutathione synthesis stimulation during in vitro maturation of ovine oocytes on embryo development and intracellular peroxide content. Theriogenology 57 1443-1451.

Downs, S, P Humpherson, and H Leese 1998 Meiotic Induction in Cumulus Cell-Enclosed Mouse Oocytes : Involvement of the Pentose Phosphate Pathway. Biol Reprod 58 1084-1094.

Dumollard, R, P Marangos, G Fitzharris, K Swann, M Duchen, and J Carroll 2004 Sperm-triggered [Ca2+] oscillations and $\mathrm{Ca} 2+$ homeostasis in the mouse egg have an absolute requirement for mitochondrial ATP production. Development 131 3057-3067.

Dunning, KR, and RL Robker 2012 Promoting lipid utilization with l-carnitine to improve oocyte quality. Anim Reprod Sci 134 69-75.

Dworkin, MB, and E Dworkin-Rastl 1989 Metabolic regulation during early frog development: glycogenic flux in Xenopus oocytes, eggs, and embryos. Dev Biol 132 512-523.

Fair, T 2010 Mammalian oocyte development: checkpoints for competence. Reprod Fert Dev 22 13-20.

Ferguson, EM, and HJ Leese 2006 A potential role for triglyceride as an energy source during bovine oocyte maturation and early embryo development. Mol Reprod Dev 73 1195-1201.

Ferreira, CR, SA Saraiva, RR Catharino, JS Garcia, FC Gozzo, GB Sanvido, LF Santos, EG Lo Turco, JH Pontes, AC Basso, RP Bertolla, R Sartori, MM Guardieiro, F Perecin, FV Meirelles, JR Sangalli, and MN Eberlin 2010 Single embryo and oocyte lipid fingerprinting by mass spectrometry. J Lipid Res 51 1218-1227.

Frank, LA, ML Sutton-McDowall, RB Gilchrist, and JG Thompson 2014 The effect of peri-conception hyperglycaemia and the involvement of the hexosamine biosynthesis pathway in mediating oocyte and embryo developmental competence. Mol Reprod Dev MRD 22299 Epub.

Frank, LA, ML Sutton-McDowall, DL Russell, X Wang, DK Feil, RB Gilchrist, and JG Thompson 2013 Effect of varying glucose and glucosamine concentration in vitro on mouse oocyte maturation and developmental competence. Reprod Fertil Dev 25 1095-1104.

Furnus, CC, DG de Matos, and DF Moses 1998 Cumulus expansion during in vitro maturation of bovine oocytes: relationship with intracellular glutathione level and its role on subsequent embryo development. Mol Reprod Dev 51 76-83.

Gendelman, M, and Z Roth 2012 Incorporation of coenzyme Q10 into bovine oocytes improves mitochondrial features and alleviates the effects of summer thermal stress on developmental competence. Biol Reprod 87118.

Gutnisky, C, GC Dalvit, LN Pintos, JG Thompson, MT Beconi, and PD Cetica 2007 Influence of hyaluronic acid synthesis and cumulus mucification on bovine oocyte in vitro maturation, fertilisation and embryo development. Reprod Fertil Dev 19 488-497.

Gutnisky, C, GC Dalvit, JG Thompson, and PD Cetica 2013a Pentose phosphate pathway activity: effect on in vitro maturation and oxidative status of bovine oocytes. Reprod Fertil Dev

Gutnisky, C, S Morado, GC Dalvit, JG Thompson, and PD Cetica 2013b Glycolytic pathway activity: effect on IVM and oxidative metabolism of bovine oocytes. Reprod Fertil Dev 25 1026-1035.

Gutzeit, HO, D Zissler, V Grau, M Liphardt, and UR Heinrich 1994 Glycogen stores in mature ovarian follicles and young embryos of Drosophila: ultrastructural changes and some biochemical correlates. Eur J Cell Biol 63 52-60.

Hashimoto, S 2009 Application of in vitro maturation to assisted reproductive technology. J Reprod Dev 55 1-10.

Hemmings, KE, HJ Leese, and HM Picton 2012 Amino acid turnover by bovine oocytes provides an index of oocyte developmental competence in vitro. Bio Reprod 86 165, 161-112.

Hocquette, JF, and D Bauchart 1999 Intestinal absorption, blood transport and hepatic and muscle metabolism of fatty acids in preruminant and ruminant animals. Reprod Nutr Dev 39 27-48.

Issaq, HJ, QN Van, TJ Waybright, GM Muschik, and TD Veenstra 2009 Analytical and statistical approaches to metabolomics research. J Sep Sci 32 2183-2199.

Kind, KL, KK Tam, KM Banwell, AD Gauld, DL Russell, AM Macpherson, HM Brown, LA Frank, DJ Peet, and JG Thompson 2014 Oxygen-regulated gene expression in murine cumulus cells. Reprod Fertil Dev RD13249 Epub.

Koek, MM, F Bakels, W Engel, A van den Maagdenberg, MD Ferrari, L Coulier, and T Hankemeier 2010 Metabolic profiling of ultrasmall sample volumes with GC/MS: from microliter to nanoliter samples. Anal Chem 82 156-162.

Kraly, JR, RE Holcomb, Q Guan, and CS Henry 2009 Review: Microfluidic applications in metabolomics and metabolic profiling. Anal Chim Acta 653 23-35.

Krisher, R 2013 In vivo and in vitro environmental effects on mammalian oocyte quality. Ann Rev Anim Bioscience $1393-417$.

Krisher, RL, and BD Bavister 1999 Enhanced glycolysis after maturation of bovine oocytes in vitro is associated with increased developmental competence. Mol Reprod Dev 53 19-26.

Krisher, RL, and RS Prather 2012 A role for the Warburg Effect in preimplantation embryo development: Metabolic modification to support rapid cell proliferation. Mol Reprod Dev 79 311-320.

Labrecque, R, and MA Sirard 2014 The study of mammalian oocyte competence by transcriptome analysis: progress and challenges. Mol Hum Reprod 20 103-116.

Leese, HJ, I Donnay, and JG Thompson 1998 Human assisted conception: a cautionary tale. Lessons from domestic animals. Hum Reprod 13 Suppl 4 184-202.

Leoni, GG, D Bebbere, S Succu, F Berlinguer, F Mossa, M Galioto, L Bogliolo, S Ledda, and S Naitana 2007 Relations between relative mRNA abundance and developmental competence of ovine oocytes. Mol Reprod Develop 74 249-257. 
Leroy, JL, T Vanholder, B Mateusen, A Christophe, G Opsomer, A de Kruif, G Genicot, and A Van Soom 2005 Non-esterified fatty acids in follicular fluid of dairy cows and their effect on developmental capacity of bovine oocytes in vitro. Reproduction 130 485-495.

Lonergan, P, and T Fair 2008 In vitro-produced bovine embryos - Dealing with the warts. Theriogenology 69 17-22.

Lonergan, P, and T Fair 2014 The ART of studying early embryo development: Progress and challenges in ruminant embryo culture. Theriogenology 81 49-55.

Luciano, AM, F Franciosi, SC Modina, and V Lodde 2011 Gap junction-mediated communications regulate chromatin remodeling during bovine oocyte growth and differentiation through CAMP-dependent mechanism(s). Biol Reprod 85 1252-1259.

Luciano, AM, S Modina, R Vassena, E Milanesi, A Lauria, and F Gandolfi 2004 Role of intracellular cyclic adenosine 3',5'-monophosphate concentration and oocytecumulus cells communications on the acquisition of the developmental competence during in vitro maturation of bovine oocyte. Biol Reprod 70 465-472.

Macmillan, KL 2007 The effect of the "Phantom Cow" Syndrome in Victorian dairy herds. Cattle Practice $\mathbf{1 5}$ 29-35.

Martino, NA, GM Lacalandra, M Filioli Uranio, B Ambruosi, M Caira, F Silvestre, F Pizzi, S Desantis, G Accogli, and ME Dell'Aquila 2012 Oocyte mitochondrial bioenergy potential and oxidative stress: within-/between-subject, in vivo versus in vitro maturation, and age-related variations in a sheep model. Fertil Steril 97 720-728 e721.

Matoba, S, L O'Hara, F Carter, AK Kelly, T Fair, D Rizos, and P Lonergan 2012 The association between metabolic parameters and oocyte quality early and late postpartum in Holstein dairy cows. J Dairy Sci 95 1257-1266.

McEvoy, TG, GD Coull, PJ Broadbent, JS Hutchinson, and BK Speake 2000a Fatty acid composition of lipids in immature cattle, pig and sheep oocytes with intact zona pellucida. J Reprod Fertil 118 163-170.

McEvoy, TG, KD Sinclair, LE Young, I Wilmut, and JJ Robinson 2000b Large offspring syndrome and other consequences of ruminant embryo culture in vitro: relevance to blastocyst culture in human ART. Hum Fertil 3 238-246.

Mirshamsi, SM, H Karamishabankareh, M AhmadiHamedani, L Soltani, H Hajarian, and AR Abdolmohammadi 2013 Combination of oocyte and zygote selection by brilliant cresyl blue (BCB) test enhanced prediction of developmental potential to the blastocyst in cattle. Anim Reprod Sci 136 245-251.

Morado, S, P Cetica, M Beconi, JG Thompson, and G Dalvit 2013 Reactive oxygen species production and redox state in parthenogenetic and sperm-mediated bovine oocyte activation. Reproduction 145 471-478.

Morado, SA, PD Cetica, MT Beconi, and GC Dalvit 2009 Reactive oxygen species in bovine oocyte maturation in vitro. Reprod Fertil Dev 21 608-614.

Nagy, ZP, S Jones-Colon, P Roos, L Botros, E Greco, J Dasig, and B Behr 2009 Metabolomic assessment of oocyte viability. Reprod Biomed Online 18 219-225.

Nel-Themaat, L, and ZP Nagy 2011 A review of the promises and pitfalls of oocyte and embryo metabolomics. Placenta 32 Suppl 3 S257-263.

Paczkowski, M, E Silva, WB Schoolcraft, and RL Krisher 2013 Comparative importance of fatty acid beta-oxidation to nuclear maturation, gene expression, and glucose metabolism in mouse, bovine, and porcine cumulus oocyte complexes. Biol Reprod $\mathbf{8 8} 111$.

Peddinti, D, E Memili, and SC Burgess 2010 Proteomicsbased systems biology modeling of bovine germinal vesicle stage oocyte and cumulus cell interaction. PLoS ONE 5 e11240.

Phongnimitr, T, Y Liang, K Srirattana, K Panyawai, N Sripunya, C Treetampinich, and R Parnpai 2013 Effect of L-carnitine on maturation, cryo-tolerance and embryo developmental competence of bovine oocytes. Anim Sci 84 719-725.

Pike, IL, and RG Wales 1982 Uptake and incorporation of glucose especially into the glycogen pools of preimplantation mouse embryos during culture in vitro. Aust J Biol Sci 35 195-206.

Pujol, M, M Lopez-Bejar, and M-T Paramio 2004 Developmental competence of heifer oocytes selected using the brilliant cresyl blue (BCB) test. Theriogenelogy $61735-744$

Redding, GP, JE Bronlund, and AL Hart 2006 The effects of IVF aspiration on the temperature, dissolved oxygen levels, and $\mathrm{pH}$ of follicular fluid. J Assist Reprod Genet 23 37-40.

Redding, GP, JE Bronlund, and AL Hart 2007 Mathematical modelling of oxygen transport-limited follicle growth. Reproduction 133 1095-1106.

Redding, GP, JE Bronlund, and AL Hart 2008 Theoretical investigation into the dissolved oxygen levels in follicular fluid of the developing human follicle using mathematical modelling. Reprod Fertil Dev 20 408-417.

Richani, D, LJ Ritter, JG Thompson, and RB Gilchrist 2013 Mode of oocyte maturation affects EGF-like peptide function and oocyte competence. Mol Hum Reprod 19 500-509.

Rieger, D 1992 Relationships between energy metabolism and development of early mammalian embryos. Theriogenology 37 75-93.

Rieger, D, and NM Loskutoff 1994 Changes in the metabolism of glucose, pyruvate, glutamine and glycine during maturation of cattle oocytes in vitro. J Reprod Fertil 100 257-262.

Robinson, JW, M Zhang, LC Shuhaibar, RP Norris, A Geerts, F Wunder, JJ Eppig, LR Potter, and LA Jaffe 2012 Luteinizing hormone reduces the activity of the NPR2 guanylyl cyclase in mouse ovarian follicles, contributing to the cyclic GMP decrease that promotes resumption of meiosis in oocytes. Dev Biol 366 308-316.

Roca, J, E Martinez, JM Vazquez, and X Lucas 1998 Selection of immature pig oocytes for homologous in vitro penetration assays with the brilliant cresyl blue test. Reprod Fertil Dev 10 479-485.

Rushmer, RA, and RL Brinster 1973 Carbon dioxide production from pyruvate and glucose by bovine oocytes. Exp Cell Res 82 252-254.

Salhab, M, S Dhorne-Pollet, S Auclair, C Guyader-Joly, D Brisard, R Dalbies-Tran, J Dupont, C Ponsart, P 
Mermillod, and S Uzbekova 2013 In vitro maturation of oocytes alters gene expression and signaling pathways in bovine cumulus cells. Mol Reprod Dev 80 166-182.

Sasseville, M, FK Albuz, N Cote, C Guillemette, RB Gilchrist, and FJ Richard 2009 Characterization of novel phosphodiesterases in the bovine ovarian follicle. Biol Reprod 81 415-425.

Schneider, JE 2004 Energy balance and reproduction. Physiol Behav 81 289-317.

Singh, R, and KD Sinclair 2007 Metabolomics: approaches to assessing oocyte and embryo quality. Theriogenology 68 Suppl 1 S56-62.

Smith, DG, and RG Sturmey 2013 Parallels between embryo and cancer cell metabolism. Biochem Soc Trans 41 664-669.

Stojkovic, M, SA Machado, P Stojkovic, V Zakhartchenko, P Hutzler, PB Goncalves, and E Wolf 2001 Mitochondrial distribution and adenosine triphosphate content of bovine oocytes before and after in vitro maturation: correlation with morphological criteria and developmental capacity after in vitro fertilization and culture. Biol Reprod 64 904-909.

Stojkovic, M, K Westesen, V Zakhartchenko, P Stojkovic, K Boxhammer, and E Wolf 1999 Coenzyme Q(10) in submicron-sized dispersion improves development, hatching, cell proliferation, and adenosine triphosphate content of in vitro-produced bovine embryos. Biol Reprod 61 541-547.

Stokes, YM, AR Clark, and JG Thompson 2008 Mathematical modeling of glucose supply toward successful in vitro maturation of Mammalian oocytes. Tissue Eng Part $A$ 14 1539-1547.

Sturmey, RG, A Reis, HJ Leese, and TG McEvoy 2009 Role of fatty acids in energy provision during oocyte maturation and early embryo development. Reprod Domest Anim 44 Suppl 3 50-58.

Sugimura, S, S Matoba, Y Hashiyada, Y Aikawa, M Ohtake, H Matsuda, S Kobayashi, K Konishi, and K Imai 2012 Oxidative phosphorylation-linked respiration in individual bovine oocytes. J Reprod Dev 58 636-641.

Sugimura, S, LJ Ritter, ML Sutton-McDowall, DG Mottershead, JG Thompson, and RB Gilchrist 2014 Amphiregulin cooperates with bone morphogenetic protein 15 to increase bovine oocyte developmental competence: effects on gap junction-mediated metabolite supply. Mol Hum Reprod MHR-13-0285.R1 EPub.

Sugiura, K, and JJ Eppig 2005 Society for Reproductive Biology Founders' Lecture 2005. Control of metabolic cooperativity between oocytes and their companion granulosa cells by mouse oocytes. Reprod Fertil Dev 17 667-674.

Sugiura, K, FL Pendola, and JJ Eppig 2005 Oocyte control of metabolic cooperativity between oocytes and companion granulosa cells: energy metabolism. Dev Biol 279 20-30.

Sugiura, K, YQ Su, FJ Diaz, SA Pangas, S Sharma, K Wigglesworth, MJ O'Brien, MM Matzuk, S Shimasaki, and JJ Eppig 2007 Oocyte-derived BMP15 and FGFs cooperate to promote glycolysis in cumulus cells. Development 134 2593-2603.

Sutovsky, P, JE Flechon, B Flechon, J Motlik, N Peynot, P Chesne, and Y Heyman 1993 Dynamic changes of gap junctions and cytoskeleton during in vitro culture of cattle oocyte cumulus complexes. Biol Reprod $\mathbf{4 9}$ 1277-1287.

Sutton-McDowall, M, R Gilchrist, and J Thompson 2010 The pivotal role of glucose metabolism in determining oocyte developmental competence. Reproduction 139 685-695.

Sutton-McDowall, ML, D Feil, RL Robker, JG Thompson, and KR Dunning 2012 Utilization of endogenous fatty acid stores for energy production in bovine preimplantation embryos. Theriogenology 77 16321641.

Sutton-McDowall, ML, RB Gilchrist, and JG Thompson 2004 Cumulus expansion and glucose utilisation by bovine cumulus-oocyte complexes during in vitro maturation: the influence of glucosamine and folliclestimulating hormone. Reproduction 128 313-319.

Sutton-McDowall, ML, RB Gilchrist, and JG Thompson 2005 Effect of hexoses and gonadotrophin supplementation on bovine oocyte nuclear maturation during in vitro maturation in a synthetic follicle fluid medium. Reprod Fertil Dev 17 407-415.

Sutton-McDowall, ML, M Mitchell, P Cetica, G Dalvit, M Pantaleon, M Lane, RB Gilchrist, and JG Thompson 2006 Glucosamine supplementation during in vitro maturation inhibits subsequent embryo development: possible role of the hexosamine pathway as a regulator of developmental competence. Biol Reprod 74 881888.

Sutton, ML, P Cetica, RB Gilchrist, and JG Thompson 2003 The metabolic profiles of bovine cumulus-oocyte complexes: effects of oocyte-secreted factors and stimulation of cumulus expansion. Theriogenology 59500.

Thomas, RE, DT Armstrong, and RB Gilchrist 2004 Bovine cumulus cell-oocyte gap junctional communication during in vitro maturation in response to manipulation of cell-specific cyclic adenosine 3',5'-monophosophate levels. Biol Reprod 70 548-556.

Thompson, JG, ACS Bell, and HR Tervit 1995 Partitioning of glucose carbon in post-compaction ovine embryos. Anim Reprod Sci 38 119-126.

Thompson, JG, M Lane, and RB Gilchrist 2007 Metabolism of the bovine cumulus-oocyte complex and influence on subsequent developmental competence. Soc Reprod Fertil Supp 64 179-190.

Thompson, JG, DG Mottershead, and RB Gilchrist 2012 Oocyte secreted factors in domestic animals. In RL Krisher (ed.), Oocyte physiology and development in domestic animals, pp. (in press). Ames, USA: Wiley Blackwell Publishing.

Thomson, JL 1967 Effect of inhibitors of carbohydrate metabolism on the development of preimplantation mouse embryos. Exp Cell Res 46 252-262.

Van Blerkom, J, M Antczak, and R Schrader 1997 The developmental potential of the human oocyte is related to the dissolved oxygen content of follicular fluid: association with vascular endothelial growth factor levels and perifollicular blood flow characteristics. Hum Reprod 12 1047-1055.

Van Hoeck, V, JL Leroy, M Arias Alvarez, D Rizos, A 
Gutierrez-Adan, K Schnorbusch, PE Bols, HJ Leese, and RG Sturmey 2013 Oocyte developmental failure in response to elevated nonesterified fatty acid concentrations: mechanistic insights. Reproduction 145 33-44.

Van Hoeck, V, RG Sturmey, P Bermejo-Alvarez, D Rizos, A Gutierrez-Adan, HJ Leese, PE Bols, and JL Leroy 2011 Elevated non-esterified fatty acid concentrations during bovine oocyte maturation compromise early embryo physiology. PLOS ONE 6 e23183.

Walsh, SW, EJ Williams, and AC Evans 2011 A review of the causes of poor fertility in high milk producing dairy cows. Anim Reprod Sci 123 127-138.

Warburg, O 1956 On the origin of cancer cells. Science 123 309-314.

Young, LE, KD Sinclair, and I Wilmut 1998 Large offspring syndrome in cattle and sheep. Rev Reprod 3 155-163. 